\title{
Annexin A5 protein expression is associated with the histological differentiation of uterine cervical squamous cell carcinoma in patients with an increased serum concentration
}

\author{
XIN LI $^{1}$, LONG CHEN ${ }^{1}$, XIU JUN LIANG ${ }^{1}$, YU FENG GAO ${ }^{1}$, XIAO JIE WANG ${ }^{1}$, \\ QIAN XU ${ }^{1}$, YONG YAN ${ }^{1}$ and FU LU GAO ${ }^{2}$ \\ ${ }^{1}$ Basic Research Institution, Chengde Medical College, Chengde; ${ }^{2}$ College of Life Sciences, \\ Hebei Normal University, Shijiazhuang, P.R. China
}

Received May 23, 2012; Accepted August 22, 2012

DOI: $10.3892 / \mathrm{mmr} .2012 .1078$

\begin{abstract}
Annexin A5 (ANXA5) is a calcium-dependent phospholipid-binding protein belonging to the annexin family and is expressed abnormally in several types of carcinoma. In the present study, ANXA5 protein expression was evaluated by western blot analysis in a series of 60 human uterine cervical squamous cell carcinomas (UCSCCs) to search for molecular alterations that may be able to serve as useful diagnostic/prognostic markers. The upregulation of ANXA5 expression was observed in 48/60 UCSCC cases (80\%), whereas a weak expression was observed in the 25 normal uterine cervical tissues. ANXA5 expression was also analyzed by immunohistochemical staining, western blot and reverse transcription-polymerase chain reaction (RT-PCR) assays of the UCSCC and uterine cervical normal tissue lesions. All dysplastic tissues showed significantly increased ANXA5 expression compared with the weak signal observed in normal epithelia. A close association was observed between the ANXA5 expression levels and the histological grade of UCSCC. Compared with moderately and well-differentiated tumors, there was a significant increase in ANXA5 expression in poorly differentiated tumors. Furthermore, ANXA5 concentrations in the blood serum of the patients were significantly increased. Our findings clearly identify ANXA5 as an effective differentiation marker for the histopathological grading of UCSCCs and for the detection of epithelial dysplasia. The results from our study support the critical role of ANXA5 in the molecular profiling of UCSCC.
\end{abstract}

Correspondence to: Professor Gao Fu Lu, College of Life Sciences, Hebei Normal University, No. 20 Road East of 2nd Ring South, Yuhua District, Shijiazhuang, Hebei 050024, P.R. China E-mail: gaofulul@yahoo.com.cn

Key words: annexin A5, uterine cervical squamous cell carcinoma, histological differentiation, serum concentration, expression

\section{Introduction}

Cervical carcinoma is the second most common type of cancer among women worldwide. More than 370,000 women are diagnosed annually with an overall survival rate of $40 \%$ (1). Of note, the high rate of mortality has remained relatively static. Although the preventive uterine cervical carcinoma (UCC) vaccine has come onto the market, it only targets human papillomavirus (HPV) which is not the sole cause of UCC. Therefore, the identification of new biological markers may be useful for predicting patient outcome.

Annexins are a group of at least 13 structurally related proteins that share a core domain containing four repeats (eight for Annexin VI) of approximately 70 conserved amino acids (2). Each of these repeats contains $\mathrm{Ca}^{2+}$ and phospholipid-binding sites. The N-terminus, specific for each annexin, contains phosphorylation sites for protein kinase $\mathrm{C}$ (PKC) as well as for other kinases. Annexins have been associated with a wide variety of functions, including membrane organization, exocytosis, endocytosis, ion channel regulation, ion channel activity and membrane-to-cytoskeleton linkage. In contrast with the others, annexin A5 (ANXA5), which is more often considered as a major intracellular $\mathrm{Ca}^{2+}$-binding protein, has a very short $\mathrm{N}$-terminus which is not phosphorylated. It is known for inducing $\mathrm{Ca}^{2+}$ influx by forming $\mathrm{Ca}^{2+}$ channels (3) and for inhibiting the activities of enzymes linked to $\mathrm{Ca}^{2+}$ activation, including phospholipase A2 and PKC (4). ANXA5 has thus been suggested to mediate $\mathrm{Ca}^{2+}$ signaling, cell cycle regulation, signal transduction, membrane trafficking and organization (5). It also binds to negatively charged phosphatidylserine (PS) with high affinity in the presence of $\mathrm{Ca}^{2+}$ ions. It has been proposed that the binding of ANXA5 to PS accounts for its anticoagulant (6), anti-apoptotic (7) and antiinflammatory (8) effects.

In the present study, using reverse transcription-polymerase chain reaction (RT-PCR), western blot analysis and immunohistochemistry, we found that the ANXA5 expression levels in uterine cervical squamous cell carcinomas (UCSCCs) were significantly higher than those in normal cervical tissues. We also found that ANXA5 levels in the blood serum of patients with UCSCC were much higher than 
those of the control group with a higher positive ratio than that of squamous cell carcinoma antigen (SCCAg). The levels of ANXA5 and SCCAg were determined to have a positive correlation by relativity analysis. The results from our study demonstrate that ANXA5 is significantly overexpressed in human UCSCC and that it is closely associated with histological differentiation, which may have implications for the diagnosis and prognosis of UCSCC.

\section{Materials and methods}

Tissue samples. Surgical tissue specimens from 60 patients with UCSCC who consecutively underwent resection of their tumors at the Affiliated Hospital of Chengde Medical College, Chengde, China, were obtained for this prospective study, following institutional review board guidelines. Informed consent was obtained from each patient. None of them had received radiochemotherapy prior to surgical intervention. A total of 25 clinically normal cervical tissues were also collected. All tissue samples were stored at $-80^{\circ} \mathrm{C}$ until analysis. A portion of the surgical tissue specimen was fixed in $4 \%$ buffered formaldehyde for use in immunohistochemical staining and the other portion was prepared for use in RT-PCR and western blot assays.

Among the UCSCCs with stage I-II disease, 18 (30\%) were well differentiated, $26(43.33 \%)$ moderately differentiated and $16(26.66 \%)$ poorly differentiated. The mean age of the patients was 57.3 (median). The stage of disease was determined following the surgical resection of the tumor according to the current tumor-node-metastasis staging (TNM) system of the International Union Against Cancer. The histological grade was determined according to the degree of differentiation of the tumor (Broders' classification).

Apparatus and reagents. The ANXA5 mouse monoclonal antibody was purchased from Sigma (St. Louis, MO, USA); the TRIzol reagent, enzyme-linked immunosorbent assay (ELISA) kit and protein assay kit were purchased from Invitrogen Life Technologies (Carlsbad, CA, USA); and the gel image analysis system and electrophoresis apparatus were purchased from Bio-Rad (Hercules, CA, USA). The digital image analysis system was obtained from Nikon (Tokyo, Japan) and the ultraviolet analyzer was from Beckman Coulter (Miami, FL, USA).

Immunohistochemical staining. The formalin-fixed, paraffin-embedded tissues were cut into 5- $\mu \mathrm{m}$ sections. The sections were deparaffinized with standard xylene and hydrated through decreasing concentrations of ethanol. Endogenous peroxidase activity was blocked by incubation with $3 \%$ (wt/vol) $\mathrm{H}_{2} \mathrm{O}_{2}$ for $10 \mathrm{~min}$ and then antigen retrieval was achieved by microwave treatment. The tissue samples were stained via an immunohistochemical method according to conventional staining procedures. Negative controls were run synchronously. For the negative control, phosphate buffer was substituted for the primary antibody. After blocking with serum, ANXA5 mouse anti-human monoclonal antibodies were applied at dilution of 1:50 and the samples were incubated overnight at $4^{\circ} \mathrm{C}$. The samples were rinsed three times with PBS ( $\mathrm{pH} \mathrm{7.2)} \mathrm{for} 5 \mathrm{~min}$ each, incubated with biotin-labeled goat anti-mouse $\mathrm{IgG}$ for $30 \mathrm{~min}$ at room temperature, rinsed again and then incubated with horseradish peroxidase (HRP)-conjugated streptavidin for $30 \mathrm{~min}$ at $37^{\circ} \mathrm{C}$. Finally, the sections were rinsed and stained with a DAB system kit. The semi-quantitative assay was conducted under a high power lens (x400) with integration of the staining intensity and the staining distribution. The ANXA5 immunohistochemical staining score was assessed using MiVnt image analysis software.

Western blot analysis. The tissues were homogenized in a single detergent lysis buffer $(50 \mathrm{mM}$ Tris, $\mathrm{pH} 8.0 ; 150 \mathrm{mM}$ $\mathrm{NaCl} ; 1 \%$ Triton $\mathrm{X}-100$; and $0.5 \%$ each of protease and phosphatase inhibitor cocktails) and then centrifuged at $12,000 \mathrm{xg}$ for $20 \mathrm{~min}$ at $4^{\circ} \mathrm{C}$. The supernatants were transferred into new tubes, assayed for protein content using a protein assay kit, aliquoted at a concentration of $5 \mu \mathrm{g} / 20 \mu \mathrm{l}$ in lysis buffer and stored at $-80^{\circ} \mathrm{C}$. Alternatively, they were used on the same day. The samples were mixed with loading buffer $(100 \mathrm{mM}$ Tris, pH 6.8; 200 mM DTT; 4\% SDS; 20\% glycerol; and 0.2\% bromophenol blue) at a 1:1 dilution, boiled for 5 min, quickly chilled on ice and then separated on $10 \%$ SDS-polyacrylamide Tris-glycine gels. The proteins were then transferred onto polyvinylidene difluoride membranes and treated with 5\% non-fat dry milk in $1 \mathrm{X}$ phosphate-buffered saline-Tween (PBST) (1.46 mM NaH${ }_{2} \mathrm{PO}_{4} \mathrm{H}_{2} \mathrm{O} ; 8.05 \mathrm{mM} \mathrm{Na}_{2} \mathrm{HPO}_{4}$; and $144.72 \mathrm{mM}$ $\mathrm{NaCl} ; 5 \%$ Tween-20) for $2 \mathrm{~h}$. The membranes were reacted with mouse anti-ANXA5 monoclonal antibodies at 1:1,000 dilution overnight at $4^{\circ} \mathrm{C}$ and then reacted with HRP-conjugated goat anti-mouse antibodies for $1 \mathrm{~h}$ at room temperature. The bound antibodies were detected by chemiluminescence according to the manufacturer's instructions and quantified using a digital image analysis system. All experiments were performed three times in triplicate.

RNA extraction and cDNA synthesis. Total RNA was prepared using the TRIzol reagent according to the manufacturer's instructions. RNA was treated with reverse transcriptase in the presence of $50 \mu \mathrm{M}$ primers in $20 \mu \mathrm{l} \mathrm{RT}$ buffer (1X Superscript II RT buffer, $10 \mathrm{mM}$ DTT and $0.025 \mathrm{mM}$ dNTP) at $25^{\circ} \mathrm{C}$ for $5 \mathrm{~min}$, followed by $50^{\circ} \mathrm{C}$ for $50 \mathrm{~min}$. Reverse transcriptase was inactivated at $70^{\circ} \mathrm{C}$ for $15 \mathrm{~min}$.

$R T-P C R$. Total RNA was isolated from the cervical cancer or normal tissues with the TRIzol reagent kit according to the manufacturer's instructions. RNA $(2 \mu \mathrm{g})$ was reverse-transcribed with 10,000 units of reverse transcriptase and $0.5 \mu \mathrm{g} / \mu \mathrm{l}$ oligo-(dT) primer. PCR amplification of the cDNA aliquots was performed with the following sense and antisense primers: ANXA5 forward, 5'-ATG GCA CAG GTT CTC AGA GGC ACTG-3' and reverse, 5'-TTA GTC ATC TTC TCC ACA GAG CAGC-3'. PCR was performed in $50 \mu \mathrm{l}$ of $10 \mathrm{mM}$ Tris- $\mathrm{HCl}$ (pH 8.3), $25 \mathrm{mM} \mathrm{MgCl}_{2}, 10 \mathrm{mM}$ dNTP, 100 units Taq DNA polymerase and $0.1 \mu \mathrm{M}$ of each primer. The reactions were initiated at $94^{\circ} \mathrm{C}$ for $5 \mathrm{~min}$ and amplified for 30 cycles of $30 \mathrm{sec}$ at $94^{\circ} \mathrm{C}, 30 \mathrm{sec}$ at $58^{\circ} \mathrm{C}$ and $30 \mathrm{sec}$ at $72^{\circ} \mathrm{C}$. Final extensions were performed for $7 \mathrm{~min}$ at $72^{\circ} \mathrm{C}$ to complete the polymerization. The PCR products were resolved on a $1 \%$ agarose gel and analyzed using a digital image analysis system. All experiments were performed at least three times, with similar results. 
ELISA. Blood samples were immersed in ice and transported immediately to a biosafety level-II laboratory for processing. Plasma was separated by centrifugation $(2,000 \mathrm{x}$ g for $10 \mathrm{~min})$ at $4^{\circ} \mathrm{C}$. The serum was extracted and stored in $300 \mu \mathrm{l}$ aliquots at $-70^{\circ} \mathrm{C}$ until analysis. The wells of 96-well Polysorb ELISA plates were coated with $50 \mathrm{ng}$ ANXA5 or SCCAg in bicarbonate buffer (0.1 M, pH 9.6). Serum samples were thawed at room temperature and $50 \mathrm{ml}$ patient serum or standard $(0$; $0.025 ; 0.050 ; 0.075 ; 0.1 ; 0.3 ; 1$ and $2.5 \mathrm{mg} / \mathrm{ml})$ were incubated with biotinylated primary antibody (dilution: $1 / 1000$ in PBS; $0.05 \%$ Tween-20; and $1 \%$ BSA) overnight at $4^{\circ} \mathrm{C}$. The plates were washed and then incubated with blocking buffer (PBS buffer $\mathrm{pH} 7.4,3 \% \mathrm{BSA}$ ) for $1 \mathrm{~h}$ at room temperature. After washing, the sample/antibody mixtures were added and the plates were incubated for $4 \mathrm{~h}$ at room temperature. The plates were washed again and incubated with streptavidin-HRP (dilution: $1 / 5000$ in PBS pH 7.4; 0.05\% Tween-20; and 1\% BSA) for $1 \mathrm{~h}$ at room temperature. After washing, the plates were incubated with the HRP substrate 3,3',5,5'-tetramethylbenzidine (TMB). The reaction was stopped with $1 \mathrm{~N} \mathrm{HCl}$ and the plates were then read at $450 \mathrm{~nm}$ in a microplate reader. The optical density at $570 \mathrm{~nm}$ (background) was subtracted. The sample concentrations were determined using a standard curve (fit type: four parameter logistic).

Statistical analysis. The western blot and RT-PCR bands were analyzed using Bandscan 5.0 software. The levels of ANXA5 and SCCAg in the cervical cancer patients and normal women were analyzed using a t-test which was also employed to analyze the expression of ANXA5 in the cervical cancer and normal tissues. For the comparison of ANXA5 expression levels between the different groups, the one-way analysis of variance test was used. The correlation between the serum concentrations of ANXA5 and SCCAg was analyzed using the Pearson correlation co-efficient. All data are expressed as the means \pm standard deviation. $\mathrm{P}<0.05$ was assumed to indicate a statistically significant difference.

\section{Results}

Expression of ANXA5 protein increased in UCSCCs. As shown in Fig. 1, ANXA5 protein expression in the 25 normal cervical tissues and the tumor tissues from 60 patients with primary UCSCC was analyzed using a commercially available mouse monoclonal antibody against ANXA5. The expression of ANXA 5 was normalized using $\beta$-actin protein. High expression levels of ANXA5 were detected in 48 of the UCSCC samples whereas only a weak expression of ANXA5 was observed in the 25 normal uterine cervical tissues. The positive rate of ANXA5 expression was $80 \%$. The data obtained from each group revealed that the expression of ANXA5 protein in the cervical cancer tissues $(0.4699 \pm 0.018)$ was significantly increased compared with that in the normal cervical tissues $(0.2023 \pm 0.022 ; \mathrm{P}<0.05)$.

To confirm the results from the western blot analysis and to investigate the presence of ANXA5 protein expression in human UCC tissues, ANXA5 expression was further examined by immunohistochemical staining. Compared with the normal epithelia, the UCSCC specimens had a markedly increased ANXA5 expression. The ANXA5 staining was preferentially

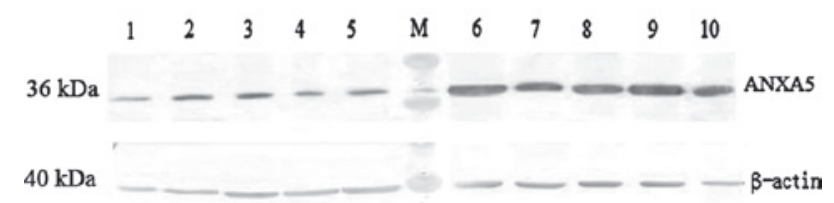

Figure 1. Increase in annexin A5 (ANXA5) protein expression in uterine cervical squamous cell carcinoma (UCSCC) determined by western blot analysis. M, protein marker; lanes 1-5, normal group; lanes 6-10, UCSCC group.

cytoplasm-localized, although nuclear and membrane staining were also noted in the superficial layers. ANXA5 expression was also detected in the normal uterine cervical tissues with a weak positive signal toward the basal cells and the most external layers of the epithelia (Fig. 2).

ANXA5 expression correlates with histological differentiation grade in UCSCC. From the immunohistochemical staining of the UCSCC tissues, we found that ANXA5 expression correlated with the histological differentiation grade of UCSCC. The images of the immunohistochemical staining showed that from well differentiated to moderately and poorly differentiated carcinoma, the number of positive cells increased and the staining of the positive cells became heavier (Fig. 3). The positive cell ratio in the poorly differentiated group was the highest $(78.2 \pm 9.21 \%)$, while that in the moderately differentiated group $(51.86 \pm 7.62 \%)$ was the second highest and that in the well differentiated group was the lowest $(39.57 \pm 4.33 \%)$. The difference between any two of the groups was significant $(\mathrm{P}<0.05)$.

From the results of the western blot and RT-PCR analyses, we also found that as the cells went from being well differentiated to poorly differentiated, the expression levels of the ANXA5 gene increased. The expression levels of the target gene mRNA were highest in the poorly differentiated group $(2.9729 \pm 0.1056)$, which were 1.504-fold higher than the relative expression levels of the normal group (1.9764 \pm 0.0899$)$. The second highest levels were in the moderately differentiated group $(2.8900 \pm 0.0552)$ and the lowest were in the well differentiated group (2.2857 \pm 0.1213 ; $\mathrm{P}<0.05$; Fig. 4). Moreover, the expression level of the ANXA5 protein in the well differentiated group was the lowest $(0.3929 \pm 0.0108)$, while the expression levels of the target protein in the moderately and poorly differentiated groups $(0.4622 \pm 0.1939$ and $0.4859 \pm 0.1567$, respectively) were significantly increased $(\mathrm{P}<0.05$; Fig. 5).

ANXA5 and SCCAg serum concentration levels increase in UCSCC patients. The serum levels of soluble ANXA5 and SCCAg were measured in the untreated UCSCC patients and the normal group using an ELISA kit. Each assay was calibrated using a standard curve according to the manufacturer's instructions. Each sample was measured in duplicate. The level of ANXA5 in the serum of the UCSCC patients was $52.77 \pm 11.54 \mu \mathrm{g} / \mathrm{ml}$ while that in normal women was $42.10 \pm 11.21 \mu \mathrm{g} / \mathrm{ml}$, which was a significant difference $(\mathrm{P}<0.05)$. The concentration of SCCAg was $17.90 \pm 7.85 \mu \mathrm{g} / \mathrm{ml}$ in the serum of the UCSCC patients and $12.87 \pm 2.35 \mu \mathrm{g} / \mathrm{ml}$ in normal women, which was also a significant difference 
A

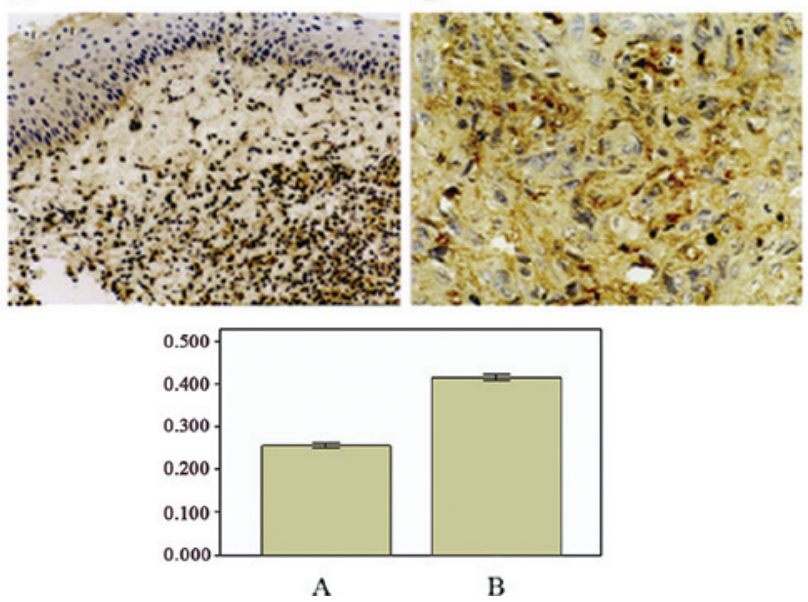

Figure 2. Annexin A5 (ANXA5) protein expression in normal uterine cervical tissues and uterine cervical squamous cell carcinoma (UCSCC) tissues by immunohistochemistry. (A) Normal uterine cervical epithelium. (B) UCSCC epithelium. Original magnification $\mathrm{x} 200$.
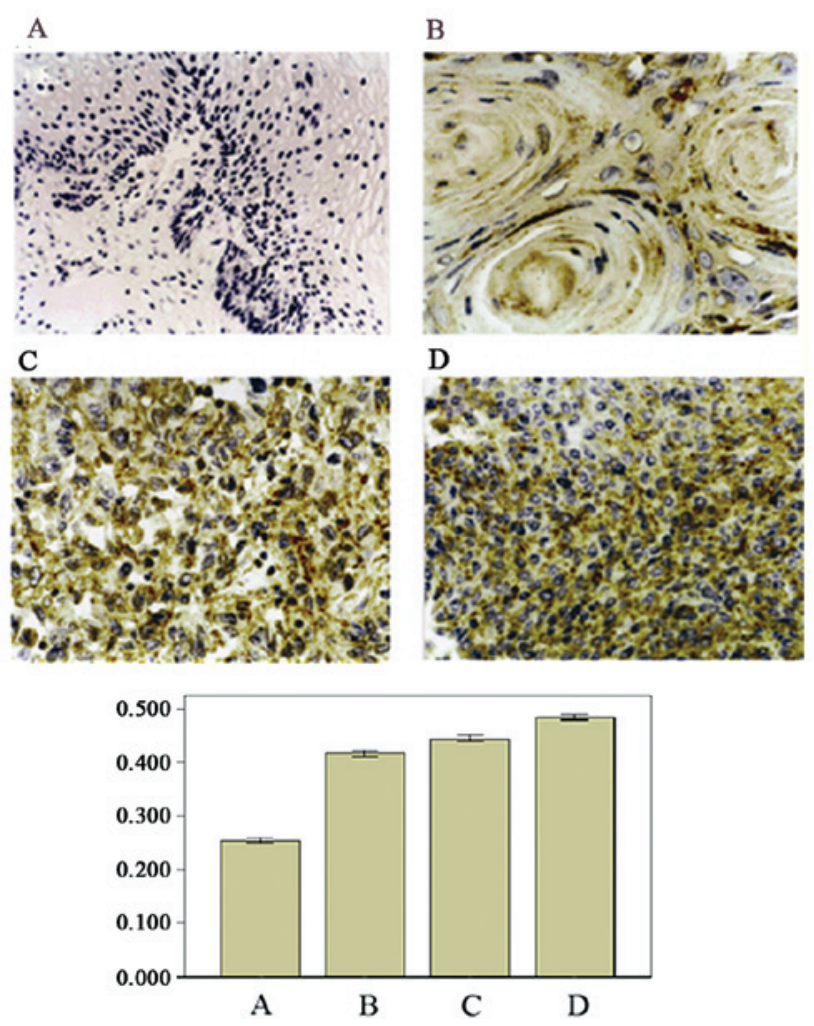

Figure 3. Immunohistochemical analysis of annexin A5 (ANXA5) for well, moderately and poorly differentiated uterine cervical squamous cell carcinoma (UCSCC). The staining of ANXA5 in (A) normal epithelium, (B) well differentiated tumor, (C) moderately differentiated tumor and (D) poorly differentiated tumor. Original magnification, (A) x200; (B-D) x400.

$(\mathrm{P}<0.05)$. The positive ratio of ANXA5 in the UCSCC patients was $83.33 \%$ while that of SCCAg was $54 \%$ which indicates that the specificity of ANXA5 was much higher than that of SCCAg.

Association of pre-treatment serum ANXA5 and SCCAg levels. SCCAg is a frequently used biomarker for squamous
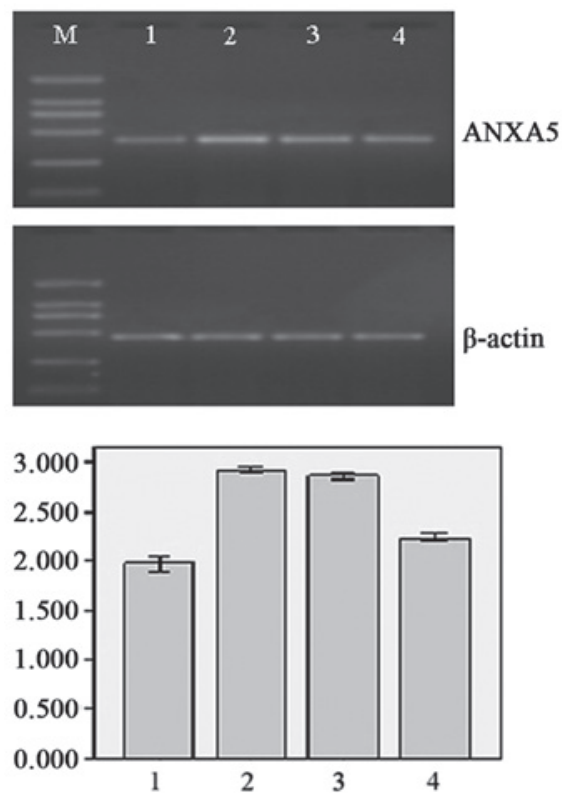

Figure 4. Expression of annexin A5 (ANXA5) mRNA by reverse transcription-polymerase chain reaction (RT-PCR). M: DNA marker; lane 1, normal uterine cervical tissue; lane 2: poorly differentiated uterine cervical squamous cell carcinoma (UCSCC); lane 3: moderately differentiated UCSCC; lane 4: well-differentiated UCSCC.
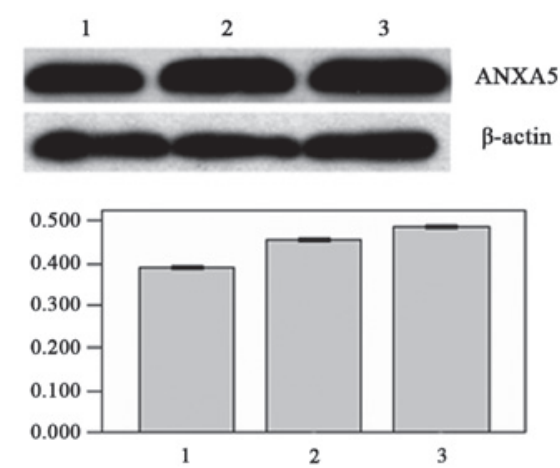

Figure 5. Annexin A5 (ANXA5) protein expression in different uterine cervical squamous cell carcinomas (UCSCCs) determined by western blot analysis. Lane 1, well-differentiated UCSCC; lane 2, moderately differentiated UCSCC; lane 3, poorly differentiated UCSCC.

cell carcinoma. To assess the correlation between ANXA5 and SCCAg, we performed Pearson correlation co-efficient analysis. SCCAg was taken as the independent variable and ANXA5 as the dependent variable. The linear regression equation was built as $\hat{\mathrm{Y}}=6.901+2.52 \mathrm{X}$ and the result demonstrated that in the UCSCC group, ANXA5 and SCCAg had a significant positive correlation $(\mathrm{r}=0.764, \mathrm{P}=0.010)$.

\section{Discussion}

Much of the complex fundamental biology of UCSCC remains poorly understood. As with other epithelial neoplasms, UCSCC appears to evolve through a multistep process involving biomolecular changes, ensuing premalignant lesions and consequent invasive cancer (9). Thus, epithelial carcinogenesis has been divided into three phases, specifically initiation, promotion 
and progression, that involve genetic alteration, dysregulated epithelial differentiation, abnormal proliferation and altered regulatory effects associated with the abnormal expression of cellular factors that regulate growth and development. The identification of molecular alterations associated with these events may yield insights into the mechanisms of initiation and progression of neoplasia and provide new tools for diagnosis, treatment and prevention

It has previously been reported that annexin A1 is able to induce differentiation in the U937 human histiocytic lymphoma cell line (10) and increasing numbers of annexin family members have been found to be involved in tumorigenesis. Based on structural characteristics, annexins have a number of physiological functions and have been confirmed to participate in numerous physiological processes. Thus, researchers have shown a keen interest in studying the role of annexins in the pathogenesis of cancer. However, it appears that the expression of different annexins is variable in various cancers and that annexins play different roles in the course of genesis of different cancers, which makes it difficult to associate their physiological functions with their behaviors in certain specific physiological processes. For example, in many cancers, including colorectal (11), gastric carcinoma (12), hepatic (13), lung (14) and pancreatic carcinoma (15), there is a sharp upregulation of several members of the annexin family at the mRNA and protein levels. However, studies have also demonstrated that in prostate cancer the levels of annexin A7 and A2 mRNA and protein are reduced (16). Although numerous hypotheses have been proposed to explain how annexins function in the genesis of different cancers, few of these hypotheses have been confirmed directly.

As a member of the annexin family, the association between tumors and ANXA5 is unclear. However, ANXA5 is highly expressed in human pituitary adenoma and growth hormone-secreting carcinoma (17) and is regarded to be one of the biomarkers in cutaneous squamous cell carcinoma due to its upregulated expression (18). In addition, ANXA5 is able to regulate the invasive capacity of oral carcinoma cells (19). As regards UCC, over a decade ago, Karube et al (20) reported that the production of ANXA5 was suppressed in carcinoma of the uterine cervix, whereas our study found that the expression of ANXA5 was increased in UCSCC at the protein and mRNA levels. This is in accordance with a study by Bae et al (21), who reported that the expression of ANXA5 was upregulated in human ICC using a 2D method. Furthermore, we detected that ANXA5 expression increased as the dysplastic epithelium became less well differentiated, which indicates that ANXA5 is associated with the differentiation of human carcinoma cells, as has been identified in human colon adenocarcinoma cells (22). Xue et al (23) reported that the expression of ANXA5 is associated with higher tumor stage and poor prognosis in colorectal adenocarcinomas.

At the time of diagnosis, the disease stage is the most important prognostic factor in the treatment of UCSCC and the identification and early treatment of small cancers correlate with excellent survival statistics. The ability to identify clinically important therapeutic targets or biomarkers for the early detection of cancer will ultimately rely on the consistency with which the protein of interest changes with respect to the population norm. Proteins with the best chances of clinical utility are those proteins whose expression patterns vary consistently, not only between different patients but also within the patient-matched sets. This consistency is likely to reflect the most important candidates for additional investigation in large validation studies. The qualitative evaluation of biological features is important for predicting the clinical course of a disease (24). Due to the histological diagnosis of UCSCC being based, until now, on the traditional examination of hematoxylin and eosin-stained specimens, supplementary techniques that use specific, disease-relevant markers may enable a more objective assessment of human uterine cervix pathology. Our findings clearly identify ANXA5 as an effective differentiation marker, providing the first demonstration of the potential utility of ANXA5 immunostaining for the histopathological grading of UCSCC and for the detection of epithelial dysplasia.

Previous studies have shown that biomarkers, including Ki-67, p53, p16ink4 and SCCAg, have some specificity for the diagnosis of UCC. However, each of them has certain limitations. To further determine the potential of ANXA5 as a diagnostic biomarker for UCSCC, we examined the levels of ANXA5 and SCCAg in the serum of the patients. The ANXA5 positive ratio was much higher than that of SCCAg and the two indexes had a positive correlation, indicating that ANXA5 could not only be used as a UCSCC biomarker by detecting serum levels but that it could also be detected with SCCAg to increase the positive diagnosis ratio.

Our results provide evidence for the increased expression of ANXA5 protein in UCSCC development. The high frequency of ANXA5 upregulation in UCSCC tumors (48 out of 60 cases) suggests that ANXA5 is fundamentally important in human tumorigenesis. Further experiments are required to establish the pathogenic role of ANXA5 in UCSCC.

\section{References}

1. Greenlee RT, Hill-Harmon MB, Murray T and Thun M: Cancer statistics. CA Cancer J Clin 51: 15-36, 2001.

2. Mortimer JC, Laohavisit A, Macpherson N, Webb A, Brownlee C, Battey NH and Davies JM: Annexins: multifunctional components of growth and adaptation. J Exp Bot 59: 533-544, 2008.

3. Camors E, Monceau V and Charlemagne D: Annexins and $\mathrm{Ca}^{2+}$ handling in the heart. Cardiovasc Res 65: 793-802, 2005.

4. Russo-Marie F: Annexin V and phospholipid metabolism. Clin Chem Lab Med 37: 287-291, 1999.

5. Gerke V and Moss SE: Annexins: from structure to function. Physiol Rev 82: 331-371, 2002.

6. Rand JH, Wu XX, Quinn AS and Taatjes DJ: The annexin A5mediated pathogenic mechanism in the antiphospholipid syndrome: role in pregnancy losses and thrombosis. Lupus 19: 460-469, 2010.

7. Kenis H, van Genderen H, Deckers NM, Lux PA, Hofstra L, Narula J and Reutelingsperger CP: Annexin A5 inhibits engulfment through internalization of PS-expressing cell membrane patches. Exp Cell Res 312: 719-726, 2006.

8. Kim DM, Noh HB, Park DS, Ryu SH, Koo JS and Shim YB: Immunosensors for detection of Annexin II and MUC5AC for early diagnosis of lung cancer. Biosens Bioelectron 25: 456-462, 2009.

9. Califano J, van der Riet P, Westra W, et al: Genetic progression model for head and neck cancer: implications for field cancerization. Cancer Res 56: 2488-2492,1996.

10. Hattori T, Hoffman T and Hirata F: Differentiation of a histiocytic lymphoma cell line by lipomodulin, a phospholipase inhibitory protein. Biochem Biophys Res Commun 111: 551-559, 1983.

11. Duncan R, Carpenter B, Main LC, Telfer C and Murray GI: Characterisation and protein expression profiling of annexins in colorectal cancer. Br J Cancer 98: 426-433, 2008. 
12. Kim JK, Kim PJ, Jung KH, et al: Decreased expression of annexin A10 in gastric cancer and its overexpression in tumor cell growth suppression. Oncol Rep 24: 607-612, 2010.

13. Zhao P, Zhang W, Tang J, et al: Annexin II promotes invasion and migration of human hepatocellular carcinoma cells in vitro via its interaction with HAb18G/CD147. Cancer Sci 101: 387-395, 2010.

14. Liu YF, Xiao ZQ, Li MX, et al: Quantitative proteome analysis reveals annexin A3 as a novel biomarker in lung adenocarcinoma. J Pathol 217: 54-64, 2009.

15. Zhang Z, Huang L, Zhao W and Rigas B: Annexin 1 induced by anti-inflammatory drugs binds to NF-kappaB and inhibits its activation: anticancer effects in vitro and in vivo. Cancer Res 70 : 2379-2388, 2010.

16. Torosyan Y, Dobi A, Glasman M, et al: Role of multi-hnRNP nuclear complex in regulation of tumor suppressor ANXA7 in prostate cancer cells. Oncogene 29: 2457-2466, 2010.

17. Mulla A, Christian HC, Solito E, Mendoza N, Morris JF and Buckingham JC: Expression, subcellular localization and phosphorylation status of annexins 1 and 5 in human pituitary adenomas and a growth hormone-secreting carcinoma. Clin Endocrinol (Oxford) 60: 107-119, 2004.

18. Dooley TP, Reddy SP, Wilborn TW and Davis RL: Biomarkers of human cutaneous squamous cell carcinoma from tissues and cell lines identified by DNA microarrays and qRT-PCR. Biochem Biophys Res Commun 306: 1026-1036, 2003.
19. Wehder L, Arndt S, Murzik U, Bosserhoff AK, Kob R, von Eggeling $\mathrm{F}$ and Melle $\mathrm{C}$ : Annexin A5 is involved in migration and invasion of oral carcinoma. Cell Cycle 8: 1552-1558, 2009.

20. Karube A, Shidara Y, Hayasaka K, Maki M and Tanaka T: Suppression of calphobidin I (CPB I) production in carcinoma of uterine cervix and endometrium. Gynecol Oncol 58: 295-300, 1995.

21. Bae SM, Min HJ, Ding GH, et al: Protein expression profile using two-dimensional gel analysis in squamous cervical cancer patients. Cancer Res Treat 38: 99-107, 2006.

22. Guzmán-Aránguez A,Olmo N, Turnay J,LeconaE,Pérez-Ramos P, López de Silanes I and Lizarbe MA: Differentiation of human colon adenocarcinoma cells alters the expression and intracellular localization of annexins A1, A2, and A5. J Cell Bioche 94: 178-193, 2005.

23. Xue G, Hao LQ, Ding FX, et al: Expression of annexin a5 is associated with higher tumor stage and poor prognosis in colorectal adenocarcinomas. J Clin Gastroenterol 43: 831-837, 2009.

24. Lomnytska MI, Becker S, Hellman K, et al: Diagnostic protein marker patterns in squamous cervical cancer. Proteomics Clin Appl 4: 17-31, 2010. 